\title{
HACIA UN ENFOQUE FILOSÓFICO-CIENTÍFICO DE LA VIDA: BIOÉTICA, CIENCIASY HUMANIDADES
}

\section{Toward a Philosophical \& Scientific Approach to Life:}

\author{
Bioethics, Sciences \& Humanities
}

ROBERTO GUTIÉRREZ LABOY

(Universidad de Puerto Rico, San Juan, Puerto Rico)

\begin{abstract}
Resumen
En este ensayo se discurre en torno al tradicional debate que se suscita cuando se confrontan las ciencias y las humanidades. Asimismo, se intenta explicar lo que realmente son las humanidades y el valor que poseen en momentos en que atraviesan la peor crisis de su historia. Después de reflexionar en torno a ese dilema, el autor considera que el conflicto entre ellas es más artificial que verdadero. Consciente que ambas áreas del conocimiento utilizan metodologías diferentes, se concluye que la una necesita de la otra. Reconociendo que otras alternativas se han empleado para solventar el problema con mayor o menor efectividad, aquí se sugiere a la disciplina de la bioética como una manera de acercarlas más. De modo que en común concierto se pueda comprender mejor el mundo y la vida que es, después de todo, el propósito fundamental tanto de los científicos como de los humanistas.
\end{abstract}

Palabras clave: Filosofía | humanidades | ciencias | bioética.

\begin{abstract}
This paper deals with the traditional debate that arises when the sciences and the humanities are confronted against each other. It also seeks to explain what the humanities actually are and their significance at a time when they are experiencing the worst crisis in its history. After reflecting upon this dilemma, the author believes that the conflict between them is more artificial than real. Even though it is clear that both areas of knowledge contain different methodologies, they need one another. Despite the fact that other alternatives have been explored to solve the problem more or less effectively, the discipline of bioethics is suggested here as a way to bring them closer. Working together we could attempt to understand better the world and the life which is, after all, the primary purpose of both scientists and humanists.
\end{abstract}

Keywords: Philosophy | Humanities | Sciences | Bioethics. 
Desde algunos puntos de vista, con las ciencias y las humanidades parece ocurrir algo similar a la correlación existente entre la fe y la razón: a través de la historia se han considerado antinómicas, lo que las ha conducido continuamente a entrar en conflicto. A estos últimos conceptos se les ha tratado de reconciliar, pero los resultados no han sido del todo eficaces. Y es que dichos términos - fe y razón- son incompatibles de-por-sí, debido a que, entre otras razones, como se suele decir "la fe es ciega" (a pesar de que San Agustín intentara demostrar lo contrario), puesto que como se expresa en la Biblia "La fe es garantía de lo que se espera; la prueba de las realidades que no se ven (Hebreos 11:1)." En tanto, la razón procura -es más exige o debe exigir- que las respuestas a las interrogantes existenciales que nos aquejan a los humanos sean "sensatas" y si no se pueden "demostrar" que cuando menos sus justificaciones sean producto de un proceso profundamente reflexivo.

En cuanto a la relación de la fe y la razón, mucha energía se ha dedicado al intento de armonizarlas o, mínimamente, acercarlas. Recordemos los loables esfuerzos de Santo Tomás de Aquino y del ya mencionado San Agustín en la época antigua y con textos como la encíclica Fides et Radio del papa J uan Pablo II y en diversos escritos del ahora papa emérito Joseph Ratzinger en la era moderna. Vale destacar el famoso debate entre J ürgen Habermas y Ratzinger cuando éste en una actitud conciliadora expresa que "yo hablaría de una correlación necesaria de razón y fe, de razón y religión, que están llamadas a purificarse y regenerarse recíprocamente, que se necesitan mutuamente y deben reconocerlo" (2006:67-68). A mí me parece que la tarea no es dable porque cuando una "está" la otra no puede "entrar". Tan es así que si extrapolamos la fe por el "mythos" (que son afectos parecidos) y la razón con el "logos" (que sí son iguales) confirmamos que, precisamente, fue del enfrentamiento de entre ambas que brotaron tanto la filosofía como la ciencia (lo que las hace - a mi parecerhermanas gemelas, aunque la filosofía surgió unos "minutos" antes). Me he estado refiriendo al paso del "mythos" al "logos". Indudablemente, con las ciencias y las humanidades el escenario es muy diferente. Por cierto, yo no coincido con la tradición de ver la filosofía como la madre de todas las ciencias, pero aún menos con el pareœr del Althusser para quien el nacimiento de la filosofía es efluvio de las ciencias (1971:41). Pese a que sí concuerdo con el 
Althusser que articuló que "Eso es ley: la filosofía siempre está unida a la ciencia" (1971:4).

Encuentro que el marco relativo a estas dos áreas del saber es distinto al punto de que creo que las ciencias y las humanidades no sólo pueden armonizarse sino que, más significativo aún, se complementan y la una no puede existir sin la otra. Me explicó. Yo soy de los que creen que un científico sin una robusta formación filosófica (entiéndase humanística) no puede aspirar a más de ser un mediocre. $\mathrm{Y}$ es que sin un pensamiento crítico, reflexivo, inquisitivo y profundamente interrogativo, que muy bien provee la filosofía, lo que harían los incipientes científicos sería repetir lo que les fue enseñado. No obstante, cuando ese científico va más allá de lo aprendido, a lo largo de su adiestramiento profesional, es que puede producir nuevos aœercamientos crítico-científicos que lo pueden conducir a generar novedosas teorías.

Sé muy bien que el método científico se rige por una serie de etapas, las cuales a grosso modo- son: la observación, la hipótesis, la experimentación, la organización, las conclusiones y la teoría o ley. Sin embargo, detrás de ese proceso tiene que haber un ente pensante que ve más allá de lo que sus colegas piensan que las cosas son. Ese ente es el científico prometedor, que lo es porque también es un filósofo en ciernes o completo. Obsérvese que no estoy hablando de la filosofía natural medievalista ni de la filosofía de las ciencias, sino de la filosofía sin más.

No estoy diciendo que la una sea igual que la otra. Lo que ocurre es que son gemelas fraternas, o bien mellizas, y no idénticas. Eso fue lo que impulsó a Albert Einstein - un científico que se acercó tanto a las humanidades- a decir que, “La relación recíproca entre epistemología y ciencia es digna de atención. Una depende de la otra. La epistemología sin contacto con la ciencia es un proyecto vacío. La ciencia sin epistemología - en el caso que sea pensable- es primitiva y confusa" (Schilpp, 1970: 683-684). ${ }^{1}$ Así, Mario Bunge -un filósofo que se aproxima tanto a las ciencias- sostuvo que "No hay por qué buscar la ciencia fuera de las humanidades, cuando lo que se requiere es encararlas en forma científica; ni hay por qué buscar la filosofía fuera de la ciencia, cuando se sabe que ésta posee sustancia filosófica" (1981: 102). De ahí que yo considere

\footnotetext{
${ }^{1}$ Esa epistemología a la que se refería Einstein acarrea el sentido inglés del término que la concibe como la rama de la filosofía que se ocupa de los fundamentos del conocimiento, incluyendo la ciencia.
} 
mezquino el debate en torno a cuál es más importante, las ciencias o las humanidades.

Sospecho que lo que ocurre es que, por lo menos recientemente, la controversia no proviene tanto ni de los científicos ni de los filósofos (quienes deberían tener clara esta cuestión) sino de aquellos que tienen una visión mercantilista, es decir lucrativa, de la educación. Y aquéllos que siendo o creyendo ser científicos, de una parte, o filósofos, de la otra, contraponen la una con la otra, en aras de "demostrar" cuál es la más significativa, no están claros en la carreras que profesan o creen profesar. Por eso es que hay quienes dicen que dado al gran desarrollo de las ciencias las humanidades han perdido pertinencia. A ellos les respondo que para que continúe ese "gran desarrollo de las ciencias" es que cada día las humanidades son más relevantes. De modo que como el tema y la controversia siguen patentes me siento obligado a terciar en el debate, por lo que en las líneas que siguen expongo algunas reflexiones y recomendaciones, ya que me parece que la bioética puede ayudar a romper con la tesitura negativa que, a veces, les dirige a la confrontación. La teoría de la "terœera cultura" que propuso C.P. Snow y desarrolló J. Brockman no creo que sea la alternativa apropiada. Asimismo, la propuesta de la "consiliencia" de E.O. Wilson tampoco lo es, aunque la versión del Stephen J ay Gould del libro The Hedgehog, the Fox, and the Magister's Pox: Mending the Gap Between Science and the Humanities (De zorros, erizos, ciencias y humanidades) me parece que posee un marco prudente. Su planteamiento es conciliatorio al decir que:

Yo también busco la consiliencia, un 'saltar juntas' de la ciencia y las
humanidades con mayor y más fructífero contacto y coherencia - pero
una consiliencia de igual consideración que respete las diferencias
inherentes, que reconozca el valor comparable pero distinto, que
entienda la absoluta necesidad de ambos campos para cualquier vida
que se precie intelectual y espiritualmente “completa” y que busque
enfatizar y promover las numerosas regiones de las coincidencias
reales y las preocupaciones comunes. (2011: 259)

Ese es el camino justo que debemos recorrer. A pesar de que ya existe una vía sencilla que nos conduce al trabajo cooperativo entre esas dos disciplinas como pronto veremos. 


\section{¿Qué es eso de las humanidades?}

El concepto de las humanidades se puede entender en dos sentidos. Primero, como un conjunto de disciplinas tales como la historia, las artes, la literatura, la filosofía y el estudio de las religiones y los idiomas. Cada una de esas materias es independiente de las otras, mas las vincula la creación cultural que producen. En las instituciones académicas se enseñan por separado. De allí las Facultades o Escuelas de Humanidades en diversos centros de educación superior. Estas "humanidades" se me antoja denominarlas de "segundo orden". Este es el entendimiento más generalizado de las humanidades. El problema con esa concepción - que no es equivocada- es que es incompleta y nos priva del entendimiento que el diálogo con la tradición humanística nos provee (Proctor, 1998: ix).

Segundo, podemos visualizar las humanidades como una disciplina en sí en la que las materias antes mencionadas se emplean de forma integral (sistémica) para, prioritariamente, reflexionar sobre el ser humano y el mundo que le rodea. Se debe resaltar que si algo identifica a esas humanidades es su carácter inter, multi y transciplinario, debido a la conjunción de diversas disciplinas en una sola materia. Es esa práctica de las humanidades la que me interesa destacar en este escrito. Me referiré a esa disciplina como de "primer orden".

La concepción de las humanidades en una disciplina como tal - de primer orden- tiene su lejano origen en la studia humanitatis renacentista y fueron lideradas en la primera mitad del siglo veinte por las universidades de Columbia y Chicago en Estados Unidos. Desafortunadamente, han ido perdiendo espacio en la mayoría de los centros de enseñanza superior del mundo, pero que paradójicamente- van encontrando su lugar en las llamadas "humanidades médicas", debido a que los centros educativos que las practican han comprendido la importancia de humanizar los profesionales de la salud. Los objetivos de las humanidades de primer orden son abarcadores. Algunos son: la concienciación de un pensamiento profundamente reflexivo en torno a valores universales como lo son la dignidad, la solidaridad y la libertad humanas. Así como el intento, a largo plazo, de provocar en el alumnado una mentalidad

plenamente cívica y humana. En pocas palabras, el objetivo de las humanidades, en el sentido que ahora las intento definir, es el ser humano, su ser y hacer. El 
ser humano en su totalidad, independientemente de su etnia. Además, la reflexión humanística procura problematizar e intentar encontrar respuestas a las grandes interrogantes que han acosado al ser humano a través de la historia. Éstas son: ¿de dónde venimos? ¿quiénes somos? ¿adónde vamos? Pregunta que perpetuadas en la pintura por Paul Gauguin van al meollo de nuestra situación existencial radicalmente problemática. Cuestionamientos que nunca se han contestado satisfactoriamente, ya que las diferentes respuestas que se han emitido muchas veces son en extremo contradictorias. Sin embargo, encararlas hace más provocativo la vida misma.

Se pretende, en las últimas décadas, agrupar tanto las humanidades de primer y segundo orden con las ciencias sociales en lo que se ha dado por llamar "ciencias humanas". Yo no veo con buenos ojos esa práctica porque si bien es correcto que todas esas disciplinas que comprenden las ciencias humanas se ocupan del ser humano, lo cierto es que su fin último no necesariamente es equivalente. Las ciencias sociales, como las ciencias naturales, se preocupan principalmente de describir el objeto de sus estudios, mientras que las humanidades se ocupan, sobre todo, de la esencia del existente, en el sentido heideggeriano (Dasein), con la intención de promover la posibilidad del sensibilizar, valorizar y humanizar al ser humano. Y ésta última afirmación no es tautológica dado al hecho de que el que se sea un ser humano no necesariamente significa que se es humano. La cualidad de lo humano se podría sintetizar en la preocupación que se muestre ante la condición por el otro y por la otra. No todo ser humano es humano, por eso nos encontramos con comportamientos inhumanos o con individuos que están deshumanizados. ¿Qué significa todo esto? Pues, que a través, preferiblemente, del método socrático (Nussbaum, 2010: 46-76) en los cursos de humanidades se va generando en el alumnado una concepción más auténtica de sí mismo, del ser humano en general y del papel que éste debe desempeñar como profesional y como ciudadano en su proceder ético y moral.

Hay muchas otras razones para destacar la importancia de las humanidades. Ejemplo de ello es el más reciente libro de Martha C Nussbaum sobre las humanidades en el que la prestigiosa filósofa norteamericana destaca la importancia de las humanidades para la democracia. Es fácil coincidir con ella, pero las humanidades son igualmente valiosas para cualquier otro sistema tanto 
político como económico como lo sería el socialismo - que en todo caso también es democrático- o, incluso, el anarquismo.

\section{La práctica en la docencia de las humanidades}

A nadie debe sorprender que la enseñanza de las humanidades de primer orden varíe conforme al docente que las administre. Ello es natural, debido a que el profesor tiende a partir desde la especificidad de sus estudios, sean éstos historia, literatura, filosofía o cualquier otro. En las líneas que siguen, comentaré brevemente uno de los posibles modelos de práctica docente de las humanidades.

El curso de Humanidades se puede visualizar como una contemplación a la trayectoria histórico-cultural de los humanos en el que se enfatiza su ser y hacer en el mundo. Pero, de mayor importancia, mientras se va generando ese miramiento se tiene que, sobre todo, reflexionar en torno a la complejidad de la existencia humana. La tarea perentoria en un curso de Humanidades es fundamentalmente el reflexionar. Hay que resaltar la imperiosa necesidad de la reflexión, porque "tenemos que estar constante y persistentemente examinando $\mathrm{y}$ volviendo a examinar todas nuestras ideas, nuestros juicios, nuestras opiniones, nuestras metas, en fin, toda nuestra visión de vida" (Gutiérrez, 2005: 14). En esa reflexión acerca del transcurrir cultural se aspira a que el educando no únicamente cavile sobre los demás, sino que se penetre a sí mismo a la manera del "conócete a ti mismo" socrático y del examinarse hacia "adentro" como nos aconsejó Unamuno. La esperanza es que en ese proceso de introspección el alumnado pueda entenderse mejor y de esa forma ubicarse con mayor solidez en el mundo que le ha tocado vivir.

Para lograr ese proceso tenemos que mirar hacia atrás, ya que, como decía Séneca, los pensadores del pasado "ninguno malgastará tus años, sino que te añadirán los suyos (2002: 53). Así que deberemos recorrer con la historia lo que fue el arte, la filosofía, la literatura, el fenómeno de las creencias religiosas y el porqué de la gran cantidad de idiomas con que nos comunicamos. Conocr las civilizaciones del pasado, sea Grecia o sea Roma, por poner sólo dos ejemplos, es más bien un pretexto para reflexionar sobre nuestra radical condición existencial en el aquí y el ahora. Es, más bien, un conocer para 
reconocernos. Robert E. Proctor lo expresa muy bien cuando indica que "El pensar se produce mejor por medio de la comparación y el contraste. $\mathrm{Si}$ solamente conoces el presente, no tienes nada con que comparar y, por tanto, no tienes manera de pensarlo. Al cortar el diálogo con el pasado, nuestra mente se contrae y se atrofia" (1998: xi). Es de por sí reprobable el abandono de los estudios de la civilización oocidental en muchas universidades en donde estudiar a Lady Gaga (2012, Trussell) es más importante que leer a Aristóteles, Voltaire o Kant. Un reciente estudio demuestra que el problema pasó de ser de grave a tan crónico que apunta a la muerte (Ricketts, et. al, 2011). El conocer los otros para reconoœrnos incita a la contemplación de nuestro interior porque después de todo no somos tan diferentes de los que nos precedieron. Los humanos, lamentable es el admitirlo, siguen siendo en el presente como lo fueron en el pasado, ni mejores, ni peores. ${ }^{1}$ En esa faena tenemos que recurrir a los clásicos. Esas obras que por ser universales, maestras e inmortales nos convidan a la reflexión. Claro que en un curso de Humanidades no podemos detenernos mucho en el gran valor literario de ellos. Más bien, se leen œomo guías para entender la vida. Si ese proceso es exitoso, entonces, el curso podría ser "una experiencia transformadora" en la vida tanto de los estudiantes como del profesor.

En esos cursos, se debe privilegiar la filosofía por ser ésta, desde mi perspectiva, la más cardinal de las humanidades y la que en algunas de sus definiciones coincide plenamente con lo que son las humanidades de primer orden. ${ }^{2}$ Esa filosofía no es la "profesional", si pudiera llamarse así, sino la filosofía cuya función es intentar desentrañar el sentido y significado de la vida. Es la filosofía para el lego que, como bien dijera otro filósofo procedente de las ciencias, Bertrand Russell:

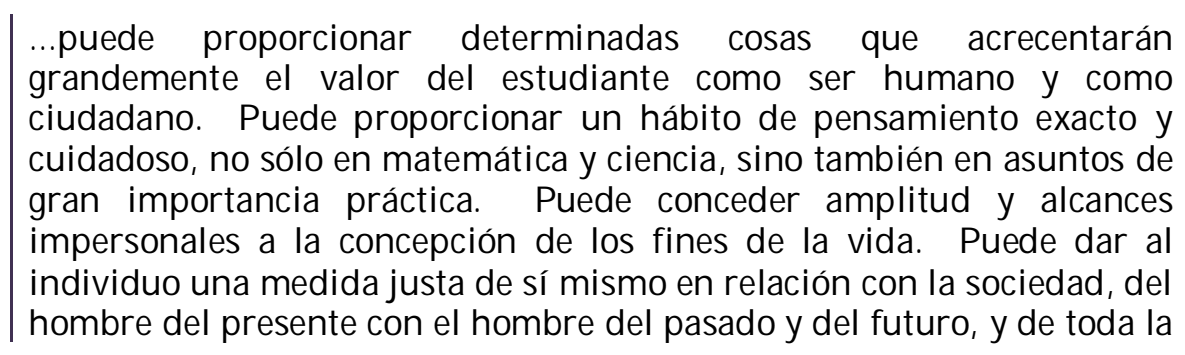

\footnotetext{
${ }^{1}$ Véase el ensayo “La inmutabilidad humana” (Gutiérrez, 2005: 49-66).

2 Escribo "algunas de sus definiciones" porque desde antigüedad se reconoce que hay tantas definiciones de filosofía como filósofos.
} 
historia del hombre en relación con el cosmos astronómico. Agrandando los objetivos de sus pensamientos, proporciona un antídoto contra las ansiedades y angustias de la hora presente y hace que se pueda acercar a la serenidad, tanto como le es posible a una mente sensible en nuestro mundo torturado e incierto. (1963: 46-47)

Esa es la filosofía que ha sido siempre pertinente para el que no se especializa en ellas. Esas son las humanidades que son fundamentales porque tienen, “...una intención formativa. Sin atribuirles poderes mágicos, ni garantizadas virtudes remediales, con ellas se aspira a crear un ámbito propicio, con su necesaria capacidad de incitación, para que, ahincados en el reconocimiento de la humana dignidad, inclinados, en reflexión, sobre nuestro mundo interior, nos adentremos alma arriba hasta las fuentes mismas del ser y el misterio de nuestro destino" (Zubiría, 1990: 496).

Einstein también nos persuade de lo relevante de esas disciplinas cuando argumenta, pese a que yo favorezco la filosofía, que:

No es suficiente enseñarle a un hombre una especialidad. A través de ella podría convertirse en una máquina útil, pero no en una personalidad armoniosamente desarrollada. Es esencial que el alumno adquiera un entendimiento y sentimiento vivo de los valores. Debe obtener un sentido vívido de la belleza de lo que es moralmente bueno. De otra manera - con sus conocimientos especializados- se asemejará más a un perro bien entrenado que a una persona armoniosamente desarrollada. Debe aprender a entender las motivaciones de los seres humanos, sus ilusiones y sus sufrimientos para poder alcanzar una relación adecuada con sus semejantes como individuos y con la comunidad. Estas valiosas cosas se transmiten a las nuevas generaciones por medio del contacto personal con aquellos que enseñan no - por lo menos no especialmente- a través de los textos. Eso es lo que principalmente forma y preserva la cultura. Es eso lo que tengo en la mente cuando recomiendo la importancia de "las humanidades" y no solamente el conocimiento seco en el campo de la historia y la filosofía. (1954: 66-67)

En mi caso, cuando comencé a enseñar humanidades hace muchos años, quizás por lo inexperto que era, proponía a los alumnos que debatiéramos sobre cuál era más importante, las ciencias o las humanidades. Ya no lo hago. Prefiero que lo descubran ellos mismos. Antes de que concluya el curso se han percatado que un adiestramiento humanístico quedaría trunco si las ciencias naturales y sociales fueran obviadas en el contexto de sus estudios profesionales, como de igual manera una formación científica sin una robusta base humanística sería un sinsentido. 
Toda esa exposición previa podría parecerle a algunos una especie de apología o justificación de las humanidades. Quizás lo sea. No obstante, me parece que las humanidades se prueban ellas mismas por la labor que han llevado a cabo a través de los siglos. Simplemente porque fomentan el pensamiento crítico, libre e integral. Sin ellas, los seres humanos quedarían reducidos a autómatas a la disposición de los dirigentes políticos y religiosos además de a las instituciones e industrias que los empleen. Es dado a ello que observo con modesta tribulación proyectos como el llamado "plan de Bolonia" cuya derrotero final será la pequeñez cultural del ser humano.

\section{¿Qué es eso de la bioética?}

El origen formal de la bioética es tan reciente como hace poco más de cuarenta años. En el inicio de la década de los setenta del recién pasado siglo, el científico norteamericano Van Rensselaer Potter formula sus cimientos en el ensayo "Bioethics: The Science of Survival" (1970) y, sobre todo, en su libro Bioethics: Bridge to the Future (1971). En realidad, en un sentido no formal, el comienzo de la bioética puede trazarse hasta, por lo menos, Hipócrates de Cos en la antigua Grecia. Yo soy de los que piensan que siempre se ha estado reflexionando filosóficamente sobre muchos de los asuntos que ahora incumben a la bioética, aunque, por supuesto, otros no existían como por ejemplo los que incumben al desarrollo de la más reciente tecnología. Empero, fue Potter quien le dio nombre a lo que actualmente denominados bioética y esbozó explícitamente los principios de esta nueva disciplina. ${ }^{1}$

La bioética, en su sentido etimológico, significa "ética de la vida". Sus postulados se dirigen a establecer el firme vínculo que debe existir entre la ética y las ciencias, particularmente en cuanto al respeto a la vida -moralmente hablando- que debe haber entre los mismos seres humanos, las plantas y los animales. En ese sentido, la bioética encuentra también parte de sus huellas iniciales en el concepto del vivir conforme a la naturaleza de los antiguos

\footnotetext{
${ }^{1}$ No paso por alto que, en 1927, el teólogo alemán Fritz J ahr empleó el término Bio-Ethik para referirse a la relación ética de los seres humanos con los animales y las plantas e, incluso, proclamó un "imperativo bioético". Ahora bien, los planteamientos de Jahr tuvieron muy poca resonancia en el mundo académico.
} 
estoicos griegos y romanos y en la muy similar concepción de vida que profesaban los pueblos autóctonos de América.

Poco después del surgir de la bioética, algunos académicos tendieron a equipararla con la ética médica hasta el punto de que muchos creen que esas actividades intelectuales son sinónimas. Eso de por sí es una visión parcializada de esa disciplina, puesto que la bioética es mucho más que eso. Lo que se puede aceptar es que la ética médica es una subdivisión de la bioética como ésta lo es de la ética y, a su vez, esta última de la filosofía. En el discurrir de este ensayo, la bioética que me interesa destacar es la potteriana. ${ }^{1}$ Motivo por el cual me concentraré en esa bioética pionera.

Fue en el año 1971 que la bioética comenzó a exhibirse como una nueva disciplina con la publicación del libro Bioética: puente hacia el futuro de Van Rensselaer Potter como antes indiqué. Su objetivo era muy claro, se proponía acercar las ciencias y las humanidades. Desde el inicio del texto, Potter deja claro que:

El propósito de este libro es contribuir al futuro de la especie humana al promover la formación de una nueva disciplina, la disciplina de la Bioética. Si hay dos culturas que parecen incapaces de comunicarse entre ellas - las ciencias y las humanidades-, y si esto es parte del motivo por el que el futuro se muestra tan inseguro, entonces deberíamos construir un puente hacia el futuro construyendo la disciplina de la Bioética como un puente entre esas dos culturas. (1971: vii)

Así que partiendo de la biología (mas sin limitarse a ella) y la ética, Potter aspiraba a establecer un diálogo en el que se dejara claro que los avances científicos no fueran a trastocar adversamente la vida como la conocemos. Las investigaciones en los campos de la biología, la medicina y la ecología debían ir mano a mano con los valores humanos.

Las definiciones que se han esbozado sobre la bioética varían de pensador a pensador. Por ejemplo, Warren Thomas Reich la considera como "el estudio sistemático de la dimensión moral -incluyendo la visión moral, decisiones, conductas y políticas- de las ciencias de la vida y del cuidado de la salud, empleando diversas metodologías en un escenario interdisciplinario"

${ }^{1}$ Para un recuento sucinto de este proceso puede consultarse a L. D. Otero (2009) y Gutiérrez (2010: 21-28). 
(Encyclopedia, 1, 1995: XXI). Mientras que Leonides Santos y Vargas nos sugiere que la:

Bioética es la disciplina que se ocupa del estudio y la reflexión en torno a la dimensión e implicaciones humanísticas y éticas de los avances en las ciencias, las profesiones de la salud y de la ecología. Es, además, una metodología de la solución racional de los problemas que surgen de las acciones y la toma de decisiones que comportan problemas y dilemas éticos en los escenarios científicos y clínicos, tanto al nivel personal como colectivo. (2006: 3 )

Yo, más bien, la concibo "como una abstracción puramente ética - es decir filosófica- y moral sobre todo aquello que producto de las ciencias, sea biología, química o física, afecta la vida del individuo, así como la interdependencia que debe existir y, en efecto, existe entre las ciencias y las humanidades" (Gutiérrez, 2012: 46). Esto implica que la bioética debe aliarse con las ciencias de forma tal que los avances científicos sean de beneficio para la humanidad y que afecten lo menos posible a los ecosistemas. Desde este punto de vista, en consecuencia, la bioética no puede limitarse a fungir como centinela del trabajo científico. De hecho, como he expresado en otra parte, la ética como tal no debe ejercer la función de preceptuar lo que es correcto o incorrecto en el proceder moral, ni de los individuos en general, ni de los científicos en particular. La ética puede exhortar, recomendar y, principalmente, promover la reflexión sobre las acciones que se lleven a cabo, pero su labor no debe ser prohibir nada (Gutiérrez, 2011). Las prohibiciones se les deja a los moralistas, quienes son los que desempeñan el triste papel de vedar acciones, la mayor parte de la veces basados en fundamentos religiosos de dudosa legitimidad. Entonces, la bioética, como una subdivisión de la ética, debe seguir ese mismo rumbo.

\section{Bioética, ciencias y humanidades}

Lo que he intentado articular más arriba tiene el objetivo de que se advierta que la bioética es parte de las humanidades, por lo menos de la forma en que la he presentado. Así que, me parece, la bioética no solamente se debe ocupar de "velar" o "custodiar" la labor de los científicos. Aún más, debe integrarse al pensamiento científico de forma y manera que los logros alcanzados por ellos sean aportaciones que propendan a un nuevo paradigma civilizatorio que 
trabaje hacia "una nueva visión de nuestro ser en el mundo y de la forma de interrelacionarnos, como fundamento de una nueva ética y política globalizadas, que tengan por objeto el bien común de la tierra y de la humanidad" (Concha, 2009).

Con todo, consideremos otra posible dimensión de esa misma disciplina. Si la bioética, en su origen, tuvo como propósito tender un puente entre las ciencias y las humanidades, pues, una vez cruzado el puente, su deber es lograr la convergencia entre esas dos disciplinas y juntas generar una visión del mundo y de la vida que subvengan en el intento de proporcionar sentido a la existencia. Esto es, la bioética además de su importante misión de convertirse en una “ciencia de la sobrevivencia” con carácter globalizador, por la que Potter abogó, nos puede muy bien proveer de mayores herramientas para lograr llevar a cabo la faena humanística de la búsqueda de lo que ha sido, es y podría ser el lugar más adecuado del ser humano en el orbe planetario. Dicho en otras palabras, las humanidades, a través de la bioética, le ha servido bien a las ciencias. De igual manera las ciencias, por medio de la bioética, han sido de mucha utilidad a las humanidades. En tanto y en cuanto los humanistas se acerquen a las ciencias podrán dilucidar y clarificar aspectos poco entendidos o mal comprendidos en torno al ser humano. Es, precisamente, a eso a lo que me refiero cuando aludo a un enfoque filosófico-científico de la vida. Téngase en cuenta que no estoy hablando únicamente de las denominadas "humanidades médicas", ya que éstas son un concepto muy restricto que sólo se ciñe a las ciencias médicas y yo me refiero a las "ciencias de la vida” como lo es la bioética según la apreciación de Potter.

Creo que aún no se le ha dado la debida consideración a este aspecto de la relación entre las ciencias y las humanidades, particularmente desde la filosofía. Por ejemplo, Pablo González Casanova ha abordado el tema, aunque sin mencionar la bioética, cuando sentencia que:

Hoy ya no podemos pensar sobre la naturaleza, la vida y la humanidad, sin tomar en cuenta los descubrimientos que se iniciaron con la cibernética, la epistemología genética, la computación, los sistemas autorregulados, adaptativos y autopoiéticos, las ciencias de la comunicación, las ciencias de la organización, las del caos determinista, los atractores y los fractales. La profundidad de esos descubrimientos va más allá de sus claras manifestaciones científicas y 
técnicas; incluye nuevas formas de pensar y actuar que comprenden las llamadas ciencias de la complejidad y las tecnociencias.

Quien no se acerque con inquietud a la comprensión y el dominio de las "nuevas ciencias" como ciencias de la complejidad no sólo no entenderá (y practicará mal) el quehacer tecnocientífico sino el artístico y el político. (2004: 11)

Hay quien sostiene que la bioética es de por sí un saber científioo. Yo no estoy seguro de que sea así. Sin embargo, sí estoy convencido que la bioética es de vital importancia para las ciencias y si ella es parte de la ética - como en efecto lo es- entonces es un conocimiento humanístico. Ese conocer auxilia a las ciencias en la medida en que le llama la atención sobre los excesos en que a menudo caen los científicos. Desde el alzar la voz para que las ciencias y la tecnología reconsideren la invención de artefactos que sean nocivos para la humanidad como la bomba atómica- hasta los muchos fraudes que se hacen en la investigación científica (Gutiérrez, “Una mirada”). Hay que tener en cuenta que el conocimiento y el poder humano han crecido como nunca antes en la historia de la humanidad, por lo que una de las funciones del humanista es sugerir que se utilice ese poder y conocimiento moral y humanamente. Pero como para recibir hay que dar, resulta que las ciencias también aportan al humanista conocimientos necesarios para, como dije, desentrañar recónditas preocupaciones humanísticas.

Prueba sustancial de esa afirmación son, entre otras, la ecología, la biología y las neurociencias. El humanista no tiene que tener la profundidad de conocimientos en esas disciplinas como la posee el especialista, pero una comprensión promedio de ellas le abrirá los ojos a la hora de intentar arrojar luminosidad en torno al lugar del ser humano en relación con el mundo y la vida. La ecología aporta a la compresión de todo el hábitat que nos rodea. ¿Cómo hemos de entender el lugar que nos corresponde en el planeta sino poseemos nociones claras sobre los ecosistemas? Un grave problema vital es que el ser humano se olvida que somos naturaleza. Por eso se define la ecología como una rama de la biología que estudia la interacción de los seres vivos con su hábitat. El conflicto estriba en que creemos que el ser humano es otra cosa cuando lo cierto es que somos parte del ecosistema. Los ecologistas y ambientalistas lo tiene muy claro y su defensa no es solamente de la fauna y la flora sino que también protegen el lugar que ocupamos en la naturaleza. Al no 
tener una clara conciencia ecológica alteramos y contaminamos el medio ambiente sin siquiera percatarnos de que nos haœmos daño a nosotros mismos. Se tuvo, entonces, que crear la ecobioética para alertar a los humanos sobre la responsabilidad que tenemos de conservar y no perturbar nuestra única morada, la Tierra. Esa concienciación es fundamental para el humanista si quiere salvar la raza humana de la autodestrucción. Además, el valor universal de la solidaridad - tan importante para las humanidades- no se debe centrar únicamente en el humano. La solidaridad, por la que tanto abogamos, tiene que existir totalmente entre los humanos, los animales y las plantas. Es ésa la base esencial de la ecología profunda de pensadores como Arne Naees, Hans J onas y Peter Singer, entre otros. Forzoso es destacar a un humanista -y precursor de la ecología- tan completo como lo fue Albert Schweitzer quien siendo filósofo y médico abogó por una filosofía basada en "la reverencia por la vida" de todos los seres. Por cierto, el Protocolo de Kioto (1997) se origina en un claro contexto ecológico y humanista.

La biología como tal - de la cual la ecología es parte- es extremadamente relevante para las humanidades en el sentido en que estudia los seres vivos como son los animales, las plantas y los propios humanos. ¿Cómo concebir filosóficamente al ser humano si desconocemos su origen, sus atributos y la constitución de su materia? El filósofo que busca comprender la esencia del ser humano precisa considerar la composición de la materia en la que habita esa esencia. De igual manera, cuando se aspira a entender al ser humano en lo que fue, es y será no se puede evitar trazar su proceso de evolución y, tal vez, su estructura celular. Conocer el proceso evolutivo de la raza humana y demás seres vivientes es fundamental no sólo para entender el pasado y el presente, es crucial para enfrentarnos a un posible futuro. Asimismo, como seres vivientes que somos, la biología nos auxilia en el mirar filosófico del existente, puesto que el entendimiento de procesos tales como los efectos positivos y adversos de la alimentación, las causas y tratamientos de las enfermedades, entre otros aspectos, nos conduce a aguzar los sentidos entorno al ser humano, por lo que no deben ser ignorados por los humanistas.

Opté por dejar para el final las neurociencias, ya que de todas las ciencias es la de mayor interés para la labor humanística y la que más controversias suscita. Las neurociencias son de por sí una práctica inter, multi y transdisciplinaria, 
dado a que en ellas se conjugan diversas áreas científicas como la química, la biología, la farmacología, la genética, etc. Su función es el estudio del sistema cerebral central de los humanos y su objetivo es, entre otros, identificar patologías cuyo origen se encuentran en las neuronas. Ello conlleva, también, el análisis científico de destrezas como el lenguaje y, de mayor relevancia, las diversas conductas humanas en relación con la configuración del cerebro y la mente. Es, justamente, de este último elemento desde donde mana una gran fuente de supuestos de mucho interés para las humanidades en general y para la ética en particular. Es mi parecer que hoy día no podemos reflexionar filosóficamente en torno al proceder humano si no tenemos en cuenta los hallazgos de las neurociencias, particularmente de la neuroética. Lo que de ninguna manera significa que serán los neurocientíficos los que pueden o deben establecer las pautas en lo concierne al procedimiento moral y conductual de hombres y mujeres, pero sí pueden ayudar enormemente.

Ahora bien, cuando se alude a la neuroética hay que precisar de qué tipo de neuroética se habla porque esta disciplina (que se desprende de la bioética) encierra dos sentidos. De una parte está la ética de la neurociencia; de la otra, la neurociencia de la ética (Roskies, 2002: 21). Como ya anticipé es la neuroética de la ética la de mayor utilidad en las reflexiones humanísticas. En un breve ensayo seminal, Roskies preconizaba que ésta "será el área con verdaderas profundas implicaciones en torno a la manera en que la ética será abordada en el siglo XXI" (2002: 22). En esa línea de pensamiento reflexiona y se cuestiona que:

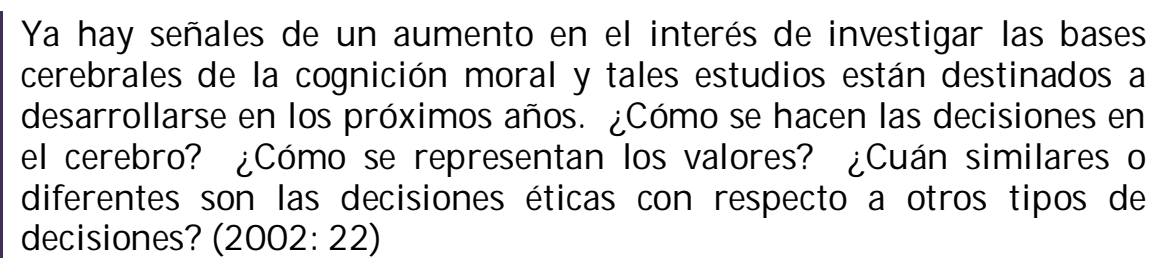

Esos planteamientos se hicieron en el año 2002, a un mes de la importante conferencia, auspiciada por la Dana Foundation, que se propusiera "trazar el campo de la neuroética" (Neuroethics: Mapping the Field). Estudios anteriores y posteriores nos invitan a replantearnos las reflexiones éticas y morales que se han producido a lo largo de los últimos veinticinco siglos en la tradición 
occidental y oriental. Antonio Damasio nos anuncia que "el error de Descartes", en cuanto a la dualidad mente-cuerpo y su famosa expresión del "pienso, luego existo", fue no haber omprendido la estrecha relación entre la emoción y la razón así como su asidero neural y químico. Revertiendo la frase cartesiana y proponiendo un "yo soy, luego pienso" (2005: 248) el afamado neurocientífico se asienta en las siguientes afirmaciones:

(1) La mente humana y el resto del cuerpo constituyen un organismo indisoluble integrado por medio de circuitos reguladores bioquímicos y neurales mutuamente interactivos (incluyendo componentes endocrinos, inmunes y neurales autonómicos).

(2) El organismo interactúa con el ambiente como un conjunto: la interacción no es ni del cuerpo solo ni del cerebro solo.

(3) Las operaciones fisiológicas que denominamos mente son derivadas de un conjunto estructural y funcional más que del cerebro solo; el fenómeno mental puede ser completamente entendido solamente en el contexto de un organismo interactuando en su medioambiente. (2005: $\mathrm{xx}-\mathrm{xxi})$

Por otro lado, Michael S. Gazzaniga, en su primer examen neuroético sacado a la luz en forma de libro nos advierte que:

La neuroética es algo más que una bioética del cerebro. A medida que se desarrolla el campo, se hace necesario ampliar sus objetivos y su ámbito. Hasta el momento, no son científicos quienes intervienen en gran parte del debate neuroético. Ya es hora de que los neurocientíficos salten a la palestra en ese campo. En mi opinión, la neuroética debe definirse como el análisis de cómo queremos abordar los aspectos sociales de la enfermedad, la normalidad, la mortalidad, el modo de vida y la filosofía de la vida, desde nuestra comprensión de los mecanismos cerebrales subyacentes. Esta disciplina no se dedica a la búsqueda de recursos para la curación médica, sino que sitúa la responsabilidad personal en un contexto social y biológico más amplio. Es - o debería ser- un intento de proponer una filosofía de la vida con un fundamento cerebral. (2005: 14-15, énfasis del autor)

Como era de esperar, de inmediato han reaocionado filósofos y filósofas ante tamaña y osada aseveración. Tal es el caso de Adela Cortina quien parece mirar con suspicacia las intenciones de los neuroéticos y exclama con cierta sorna:

Ahora bien, con las debidas cautelas podemos decir que si entendemos por "neuroética" la ética de la neurociencia, entonces es una dimensión 
de la bioética, una ética aplicada más, que da por buenas las teorías éticas existentes y trata de encontrar respuestas desde ella a problema concretos. Pero si tomamos el vocablo en la segunda acepción, como neuroética de la ética, entonces parecemos estar anunciando una auténtica revolución, porque la neurociencia nos proporcionaría el fundamento cerebral para una ética normativa, el conocimiento de los mecanismos cerebrales nos permitiría por fin aclarar científicamente qué debemos hacer moralmente. Con lo cual, como se ha dicho en alguna ocasión, los filósofos quedaríamos condenados al paro. (2010: 132)

La distinguida filósofa española prosigue planteando que "Quedarían entonœes arrumbadas, por obsoletas, las viejas éticas filosóficas y las morales religiosas y podrían sustituirse por una ética basada en la neurociencia, que sería por eso mismo universal" (2010: 133). Y, arremete tajantemente contra esa disciplina al decir que "el neurocientífico no suele molestarse en estudiar la dimensión filosófica de los problemas a los que hace frente, con lo cual acaba diciendo atrocidades sin cuento porque ni sabe de lo que habla" (2010: 144).

La apreciación de Cortina, que en otros alegatos de su ensayo contiene mucha sabiduría, es la postura equivocada ante los esfuerzos de esos estudiosos, debido a que los neurocientíficos no pretenden ser filósofos, simple y llanamente porque no lo son. Si alguno de ellos pretende presentarse como tal estaría en la senda hacia el fracaso. Dejemos que las teorías neuroéticas nos auxilien en la reflexión propiamente ético-filosófica. Creo que es, justamente, allí en donde los filósofos -y humanistas en general- pueden crecerse y no sentirse amenazados. El entender esa área del conocimiento nos puede conducir - a los que nos dedicamos a las humanidades, específicamente a la ética- a conjeturar otras alternativas ante la pregunta crucial que debemos hacernos: ¿Cuán efectiva ha sido la ética desde su inicio hasta la actualidad? Esto es, ¿después de más de veinticinco siglos de propuestas éticas y morales, tanto en el mundo occidental como en el oriental, el ser humano es ahora mejor por la influencia que las mismas sobre él han ejercido? (Gutiérrez, 2008: 15). Mucho tiene que decirnos la neuroética de la ética. Si reconocemos sus hipótesis y teorías, nuestras respuestas podrían ser más eficaces. 


\section{Una reflexión final}

El acercamiento de las humanidades a las ciencias no promueve que los que la practican piensen como científicos, como la aproximación de las ciencias a las humanidades no implica que los científicos piensen como humanistas, pese a que sería una gran cosa si fuera así en unos y otros. Lo sustancial es que las investigaciones científicas pueden ayudar a los humanistas a comprender mejor a los seres humanos y la vida así como las humanidades proveen una excelente formación cultural e inquisitiva a los científicos que mucho la necesitan. Por eso es que en la bioética como disciplina es donde la ciencia y las humanidades conviven de ejemplar manera. Por eso encontramos destacados bioéticos como Daniel Callahan que se formó como filósofo y Diego Gracia como médico, o a H. Tristram Engelhardt Jr. quien se graduó como filósofo y médico y a Volnei Garrafa quien estudió odontología, entre muchos otros ejemplos en semejante situación.

La reflexión humanística, entiéndase filosófica, nunca será desplazada por las ciencias. Ambas tienen y deben mantener su propio espacio. Ambas deben estar en continua comunicación. Si lo logran, ambas se fortalecerán. De modo que debe continuarse un diálogo franco y abierto entre ellas. Cada una debe comprender su importante lugar (espacio) y aceptar que ninguna es superior a la otra. En ese particular la bioética ha aportado elementos enjundiosos. El argumento sobre cuál es más significativa y superior, es similar a la necedad de pretender establecer cual etnia es más prominente que otra. Para el humanista como para el científico no hay diferencias valorativas entre las razas, pensar otra cosa sería sentirse inferior. El ser humano es solamente eso, humano. Фा

\section{Referencias}

ALTHUSSER, Louis. (1971). Lenin and Philosophy and other Essays. Translated by Ben Brewster. New York: Monthly Review Press. BUNGE, Mario. (1981). La ciencia: su método y su filosofía. Buenos Aires: Siglo Veinte. 
CONCHA, Miguel. (11 de julio de 2009). "Nuevo paradigma civilizatorio". La jornada, recuperado de http://www.jornada.unam.mx/2009/07/11/opinion/ 013a1pol

CORTINA, Adela. (Enero-junio 2010). "Neuroética: ¿Las bases cerebrales de una ética universal con relevancia política?” Isegoría. Revista de Filosofía Moral y Política, 42, 129-148.

DAMASIO, Antonio. (2005). Descartes' Error: Emotion, Reason, and the Human Brain. New York: Penguin Books.

Encyclopedia of Bioethics. (1995). Ed. Warren Thomas Reich. 5 vols. New York: Simon and Schuster/ Macmillan.

EINSTEIN, Albert. (1954). Ideas and Opinions. New York: Crown Publishers. GAZZANIGA, Michel S. (2005). The Ethical Brain. New York: Dana Press. GONZÁLEZ CASANOVA, Pablo. (2004). Las nuevas ciencias y las humanidades. De la academia a la política. Barcelona: Anthropos.

GOULD, Stephen J ay. (2011). The Hedgehog, the Fox, and the Magister's Pox: Mending the Gap Between Science and the Humanities. Massachussetts: Harvard University Press.

GUTIÉRREZ LABOY, Roberto. (2005). La fragilidad humana y otros ensayos: Reflexiones humanísticas. Madrid: Ediciones Atlantis.

(2008). Ética a Ana Laura: Hacia una ética humanista. Madrid: Editorial Manuscritos.

(2010). Eugenio María de Hostos: Precursor de la bioética en América Latina. Santo Domingo: Biblioteca Nacional Pedro Henríquez Ureña.

(2011). “El problema de la prohibición en la ética." Problemata: Revista Internacional de Filosofia, 2.2 156-175.

(2013). “Una mirada filosófica a la ética de la investigación." Revista Bioética, 21.143-52.

NUSSBAUM, Martha C. (2010). Not for Profit: Why Democracy Needs the Humanities. NewJ ersey: Princeton University Press.

OTERO, Luis Daniel. (Enero 2009). "Bioética: El concepto relegado.” Revista Interciencia, 34.171-76.

POTTER, Van Rensselaer. (1971). Bioethics: Bridge to the Future. New J ersey: Prentice-Hall. 
PROCTOR, Robert E. (1998). Defining the Humanities: How Discovering a Tradition Can Improve our Schools. 2 ed. Bloomington: Indiana University Press.

RATZINGER, Joseph, Jürgen Habermas. (2006). Dialéctica de la secularización: Sobre la razón y la religión. Madrid: Ediciones Encuentro.

RICKETTS, Glenn., Peter W. Wood, Stephen H. Balch y Ashley Thorne. (2011). The Vanishing West: 1964-2010, The Disappearance of Western Civilization from the American Undergraduate Curriculum. New Jersey: National Association of Scholars.

ROSKIES, Adina. (2002). "Neuroethics for the New Millennium”. Neuron, 35 21- 23

RUSSELL, Bertrand. (1963). Ensayos impopulares. México: Hermes.

SANTOS Y VARGAS, Leonides. Bioética crítica. (2006). San Juan: Instituto Hostosiano de Bioética; Decanato de Asuntos Académicos, Recinto de Ciencias Médicas, Universidad de Puerto Rico,.

SÉNECA, Lucio Anneo. (2002). De la brevedad de la vida. 4 ed. Río Piedras: Editorial dela Universidad de Puerto Rico.

SCHILPP, Paul A. (ed.) (1970). Albert Einstein: Philosopher-Scientist. New York: Library of Living Philosophers.

TRUSSELL, Tait. (January 23, 2012). “Universities Abandon Western Civilization." FrontPage Magazine. Recuperado de, http:// frontpagemag.com/2012/tait-trussell/universities-abandon-westerncivilization/

ZUBIRÍA, Ramón de. (1990). "Acerca del concepto de las humanidades." Thesaurus: Boletín del Instituto Caro y Cuervo, XLV.2 489-496 